\title{
BMJ Open Physical activity and sedentary activity: population epidemiology and concordance in Australian children aged 11-12 years and their parents
}

\author{
François Fraysse, ${ }^{1}$ Anneke C Grobler, ${ }^{\circledR 2,3}$ Josh Muller, $^{2}$ Melissa Wake, ${ }^{\odot, 3,4}$ \\ Timothy Olds ${ }^{\odot 1}$
}

To cite: Fraysse F, Grobler AC, Muller J, et al. Physical activity and sedentary activity: population epidemiology and concordance in Australian children aged $11-12$ years and their parents. BMJ Open 2019;9:136-146. doi:10.1136/ bmjopen-2018-023194

- Prepublication history and additional material for this paper are available online. To view these files, please visit the journal online (http://dx.doi. org/10.1136/bmjopen-2018023194).

Received 5 April 2018 Revised 21 January 2019 Accepted 4 April 2019

Check for updates

(C) Author(s) (or their employer(s)) 2019. Re-use permitted under CC BY-NC. No commercial re-use. See rights and permissions. Published by BMJ.

${ }^{1}$ Sansom Institute, Alliance for Research in Exercise, Nutrition and Activity (ARENA), University of South Australia, Adelaide, South Australia, Australia ${ }^{2}$ Murdoch Children's Research Institute, Parkville, Victoria Australia

${ }^{3}$ Department of Paediatrics, The University of Melbourne, Parkville, Victoria, Australia ${ }^{4}$ Department of Paediatrics and The Liggins Institute, The University of Auckland, Auckland, New Zealand

Correspondence to Professor Melissa Wake; melissa.wake@mcri.edu.au

\section{ABSTRACT}

Objectives To describe the epidemiology and parent-child concordance of objectively measured physical activity in a population-based sample of Australian parent-child dyads. Design Cross-sectional study (Child Health CheckPoint) nested within the Longitudinal Study of Australian Children. Setting Assessment centres in seven Australian cities and eight regional towns or home visits; February 2015-March 2016.

Participants of all CheckPoint families ( $n=1874), 1261$ children (50\% girls) and 1358 parent (88\% mothers) provided objectively measured activity data, comprising 1077 parent-child dyads.

Outcome measures Activity behaviour was assessed by GENEActiv accelerometer. Duration of moderateto-vigorous physical activity (MVPA) and vigorous physical activity and sedentary behaviour (SB) were derived using Cobra custom software, along with MVPA SB fragmentation and mean daily activity. Pearson's correlation coefficients and linear regression estimated parent-child concordance. Survey weights and methods accounted for the complex sample design and clustering. Results Although parents had average lower accelerometry counts than children (mean [SD] 209 [46] vs 284 [71] g.min), 93\% of parents met MVPA daily duration guidelines on published cutpoints (mean [SD] 125 [63] min/day MVPA), compared with only $15 \%$ of children (mean 32 [27] min). Parents showed less daily SB duration (parents: 540 [101], children: 681 [69] minutes) and less fragmented accumulation of MVPA (parents: $\alpha=1.85$, children: $\alpha=2.00)$. Parent-child correlation coefficients were 0.16 (95\% Cl 0.11 to 0.22$)$ for MVPA duration, 0.10 (95\% Cl 0.04 to 0.16) for MVPA fragmentation, 0.16 (95\% $\mathrm{Cl} 0.11$ to 0.22$)$ for SB duration and $0.18(95 \% \mathrm{Cl} 0.12$ to 0.23) for SB fragmentation.

Conclusions Standardised cutpoints are needed for objective activity measures to inform activity guidelines across the lifecourse. This may reflect large amounts of time in non-shared environments (school and work).

\section{INTRODUCTION}

Physical activity (PA) and sedentary behaviour (SB) have both been independently linked to a wide range of health outcomes in children ${ }^{12}$
Strengths and limitations of this study

- This study used valid, reliable, objective, free-living measures of Australian children and parent activity patterns. The sample is drawn from a nationally representative cohort.

- We report for the first time parent-child concordance in objective activity duration and fragmentation.

- Although the accelerometry measurements were objective, the multiple choices needed in processing the data could have impacted on the results, requiring caution in comparisons of absolute values with other studies.

- Findings apply to a narrow child age range (11-12 years); parent-child concordance could evolve as children grow up.

- Most adults were mothers, limiting conclusions for fathers and for adults who are not parents.

and adults. ${ }^{34}$ Furthermore, some studies have suggested that, independent of duration, other characteristics of PA and/or SB impact on health. For this reason, there has recently been a growing interest in examining the patterns of accumulation of sedentary and physical activity times, the term 'pattern' encompassing notions such as sequencing, timing, consistency and fragmentation. Recent studies have shown that less fragmentation of sedentary time (more long bouts) is associated with obesity and health markers in adults, although analyses involving children have been less conclusive. ${ }^{56}$ Similarly, higher intensity, ${ }^{7}$ more continuous ${ }^{8}$ bouts of PA have been associated with better health outcomes and most PA guidelines contain recommendations regarding the distribution of $\mathrm{PA}^{9}{ }^{10}$ Finally, vigorous PA (VPA) has added benefits compared with overall moderate-to-vigorous physical activity (MVPA), ${ }^{1}$ and some PA guidelines ${ }^{10}{ }^{11}$ provide recommendations on the amount of VPA in addition to MVPA recommendations. 
Patterns of activity and sitting result from both genetic and environmental factors, ${ }^{12}$ so we would expect a degree of concordance between children's activity patterns and those of their parents, arising from shared genes and shared environments. Shared environments include geographical, climatic and financial contexts and social factors such as parental modelling and direct parental involvement. Child-to-parent effects may also play a role. Genetic factors may relate to the heritability of personality traits associated with adherence to PA (conscientiousness, self-motivation and self-discipline), reward-associated hormonal responses to exercise (dopamine and endogenous opioids) or physiological characteristics such as aerobic fitness and strength that encourage participation in sport.

While a high parent-child concordance may be a marker of strong genetic or shared environmental determinants, a lower correlation may indicate greater importance for the non-shared environment-notably the school environment for children and the work environment for parents. School in particular may be a homogenising influence, since at school all children have very similar timings of daily activities. In terms of interventions, a high concordance would either suggest that interventions may be ineffective (if there is a large non-modifiable genetic component) or that the focus should be on the shared environment. A low concordance may be a marker of relatively high behavioural malleability, with an appropriate focus on the non-shared environment.

Advances in wearable technology have made it possible to objectively measure $\mathrm{PA}$ and $\mathrm{SB}$, and a number of studies have quantified free-living activity in children ${ }^{12-14}$ and adults. ${ }^{14-16}$ Estimates of the proportion of variability in measured PA that can be ascribed to additive genetic effects range from $20 \%$ to $71 \% .^{12} 17$ The differences may be due to the age of the participants, the powerful effect of the shared school environment or to the use of questionnaire data.

To review current literature on parent-child concordance in PA and $\mathrm{SB}$, we used a systematic search to synthesise data from 26 studies ${ }^{17-40}$ from 11 mainly European and North American countries. This yielded a total of 119 correlations between parental and child PA (figure 1). Correlations were classified according to: (1) the type of PA measured (sport, exercise, VPA, moderate PA [MPA], recreational PA, leisure-time PA were all classified as MVPA); estimates of overall energy expenditure were classified as total daily energy expenditure (TDEE); (2) the age of the child; (3) the sex of the parent; (4) the sex of the child; and (5) assessment methodology (questionnaires were classified as subjective; accelerometry, pedometry and direct observation as objective). Using a random effects approach, the overall weighted mean correlation for all PA outcomes was 0.18 (95\% confidence level [CL] 0.15 to 0.21 ) (figure 1). Correlations did not differ by outcome (MVPA: $r=0.18$, TDEE: $r=0.26$ ), sex of parent (father: $r=0.23$, mother: $r=0.18$ ), sex of child (daughter: $r=0.20$, son: $r=0.23$ ) or assessment

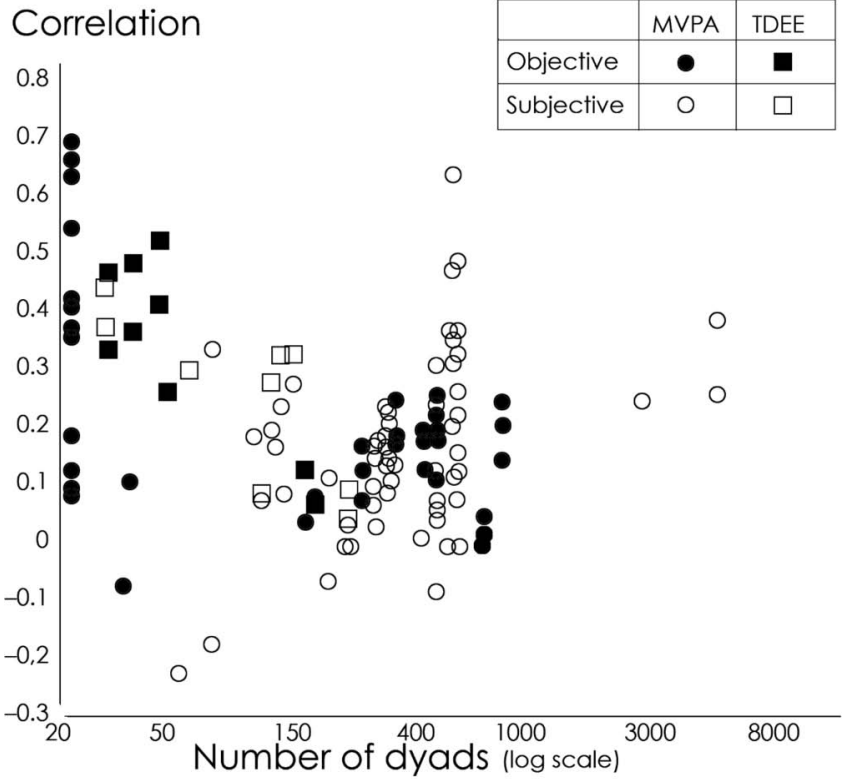

Figure 1 Funnel plot of correlations between parent and child physical activity from 26 studies. Data derived from objective measures are shown with filled symbols; data derived form subjective measures with empty symbols. Circles indicate studies where the outcome was MVPA, and squares studies where the outcome was TDEE. MVPA, moderate to-vigorous physical activity; TDEE, total daily energy expenditure.

methodology (subjective: $\mathrm{r}=0.20$, objective: $\mathrm{r}=0.17$ ). Heterogeneity was high for all analyses $\left(\mathrm{I}^{2}>55\right)$. Only four studies ${ }^{19} 212237$ from the UK, Finland and the USA, with a total of 24 correlations, looked at parent-child concordance in some measure of SB (sitting, TV or inactivity). The overall weighted mean correlation was $0.26(0.17-$ $0.35)$. Heterogeneity was high $\left(\mathrm{I}^{2}=72\right)$.

The sample sizes in most of the PA and SB studies were relatively modest (median $\mathrm{n}=192$ ), and in only 7 of 26 studies were the activity patterns of both parent and child objectively measured. None of the studies was performed in Australia. Furthermore, these studies only addressed parent-child concordance in the duration of $\mathrm{PA}$ or $\mathrm{SB}$, or total daily activity levels, with no data on fragmentation.

The aims of this study were to:

- Report the mean values and distributions of PA and $\mathrm{SB}$, and their fragmentation, in a large, population-based sample of Australian children aged 11-12 years and Australian midlife adults (their parents).

- Quantify parent-child concordance in objectively measured PA and SB duration and fragmentation. In addition, report mother-child and father-child concordances separately in order to allow comparison with previous concordance studies.

\section{METHODS}

\section{Study design and participants}

The initial study design and recruitment have been described in detail elsewhere. ${ }^{41}{ }^{42}$ The Longitudinal Study of Australian Children (LSAC) commenced in 
2004, recruiting a nationally representative cohort of 5107 infants through a two-stage cluster sample design, ${ }^{43}$ whereby $10 \%$ of all Australian postcodes were randomly selected, stratified by state and capital city/rest of state, and children born between March 2003 and February 2004 were then randomly selected from the Medicare database. $^{44} 45$ A percentage of $73.7(\mathrm{n}=3764)$ of participants were retained to LSAC wave 6 in 2014.

At the start of wave 6 , all contactable and consenting families were invited to consent to their contact details being shared with the Child Health CheckPoint team $(\mathrm{n}=3513)$. In 2015, consenting families were sent an information pack via post and received an information and recruitment phone call. The CheckPoint study was conducted from February 2015 to March 2016, between LSAC waves 6 and 7 (children's age 11-12 years), and 1874 families participated. The overall aim of the CheckPoint study was to examine relationships between multiple environmental factors and multiple health outcomes; a more detailed description of the study design is provided elsewhere. ${ }^{4647}$

\section{Consent}

The attending parent provided written informed consent for them and their child to participate in the study.

\section{Patient and public involvement}

No patient groups were involved in the design or conduct of LSAC, a population-based longitudinal study. To our knowledge, the public was not involved in the study design, recruitment or conduct of the LSAC study or its CheckPoint module. Parents received a summary health report for their child and themselves after the assessment visit. They consented to take part knowing that they would not otherwise receive individual results about themselves or their child.

\section{Procedure}

All measures were collected at a specialised 3.5-hour (seven capital cities and larger regional towns) or 2.5-hour (eight smaller regional centres) CheckPoint assessment centre visit. Three hundred and sixty-five families who could not attend a centre received a 1.5-hour home visit. At the end of the visit, a trained research assistant fitted a GENEActiv accelerometer (Activinsights Ltd, UK) on the non-dominant wrist of each child and parent and provided each with an activity card (see below). Participants were instructed to wear the device at all times for eight continuous days, starting the day of the visit, removing it only for prolonged water immersion (swimming and bath) or as prescribed by some contact sports rules (eg, netball). After 8 days, participants returned the device, together with the completed activity card using the prepaid postal envelope provided. For more information on data collection, refer to the physical activity section of the Child Health CheckPoint Data Issues Paper. $^{48}$

\section{PA measures}

Activity cards: the activity cards were paper-based logs in a table format with fields for each day of the monitoring period to allow participants to write the following:

1. At what time they went to bed at night ('bed time').

2. At what time they woke up in the morning ('get up time').

3. If they took the device off, at what times it was removed and put back on, as well as the reason for removal.

4. A brief description of their day (eg, 'school', 'travel' and 'unwell resting'...).

Once returned, activity cards were transcribed in electronic form by research assistants to be used in the processing of the accelerometer data (see below for details). Reliability testing of card transcription is described in online supplementary appendix 1 .

Accelerometers were configured through the manufacturer's software (GENEActiv PC Software, Activinsights, UK) to record at $50 \mathrm{~Hz}$ for 14 days, starting at midnight following the CheckPoint visit. The 14-day recording duration was chosen to ensure enough valid days were recorded in case the participant could not wear the device for some days and the total monitoring duration had to be extended.

After the device was returned, the research team downloaded the raw acceleration data. The signal vector magnitude of the acceleration, minus gravity, was computed and summed over 60 s epochs:

$$
S V M=\sum_{60 . s}\left|\sqrt{a_{X}^{2}+a_{Y}^{2}+a_{Z}^{2}}-g\right|
$$

where $a_{x}, a_{y}$ and $a_{z}$ are the three components of the acceleration signal and $\mathrm{g}$ the acceleration of gravity $\left(9.81 \mathrm{~m} / \mathrm{s}^{2}\right)$. The $60 \mathrm{~s}$ epoch data was then imported into custom Matlab software for further processing. This software (Cobra, developed at the University of South Australia) provides a user-friendly graphical user interface for processing accelerometer data.

First, sleep was identified using the activity cards completed by the participants. Sleep times were corrected by visual inspection when necessary, that is, in case sleep times were not reported or when obvious discrepancies were observed between reported sleep and accelerometer trace. Following this, device removals (non-wear) were identified using the activity cards and excluded from analysis. Where the reason given for removal was 'sport', the removal period was replaced with a period of MVPA. This was done because: (1) most children were not allowed to wear the watch for some sport activities (eg, netball and swimming) and (2) these sport activities often made for a large part of daily MVPA, so ignoring them would potentially result in a large underestimation of daily MVPA. MVPA imputed in this way was not considered for the calculation of MVPA fragmentation (see below). Duration of VPA was also obtained for each participant.

A day was considered invalid and excluded from analysis if it included $\leq 10$ hours wear during waking hours, ${ }^{14}$ 
or if it included $\geq 1000 \mathrm{~min}$ ( 16 hours $40 \mathrm{~min}$ ) of sedentary time (reflecting a day of non-wear not captured by the self-report logs, typically after the end of the recording period). A participant was considered invalid and excluded from analysis if they provided $<4$ valid days of accelerometry data $^{1449}$ or if they had $\leq 200 \mathrm{~min}$ average sleep time.

Each $60 \mathrm{~s}$ epoch of waking wear time was then classified into one of four PA levels: sedentary, light, moderate or vigorous PA. Cutpoints for PA levels were defined according to Esliger $e t a \tilde{l}^{\tilde{0}}$ for parents and Phillips $e t a \tilde{l}^{\tilde{1}}$ for children and adjusted proportionally to account for the $50 \mathrm{~Hz}$ sampling frequency. The resulting cutpoints between sedentary and light, light and moderate and moderate and vigorous PA were 188, 403 and 1131 gravity units per minute (g.min) for adults, and 244, 788 and 2175 g.min for children, respectively.

Fragmentation of sedentary and MVPA time was characterised using the method described by Chastin and Granat. ${ }^{52}$ The measure of fragmentation $(\alpha)$ was the slope of the regression line of the relative frequency of a bout (of MVPA or SB) plotted against bout length on a $\log$ scale. For SB, $\alpha$ was calculated on a per-day basis. However, $\alpha$ for MVPA was calculated using all valid days combined for a given participant, because a good curve fit required more bouts of MVPA than were usually available in a single day. In the present study $\alpha$ was multiplied by -1 so that it is always positive. Higher values of $\alpha$ indicate greater fragmentation, that is, fewer long boutsconsidered desirable for $\mathrm{SB}$-and lower values of $\alpha$ less fragmentation and more prolonged bouts.

\section{Other sample characteristics including potential confounders}

Age and sex affect PA patterns, which in turn were expected to influence parent-child correlations. Sex and date of birth were exported from Medicare Australia's database at the time of LSAC enrolment (for the child) or self-reported (parent). Age was rounded to nearest week by calculating the days between the participant's date of birth and date of assessment.

Adjustment was also made for socioeconomic status because it is shared by parents and children and is correlated to PA and SB patterns. Socioeconomic status was determined from the postcode of the participant's primary address using the Socio-Economic Indicators for Areas 2011 Index of Relative Social Disadvantage (Disadvantage Index), which factors in household education levels, income, employment status and disability. The population mean score for Australia is 1000 (SD 100), with higher scores representing greater advantage.

\section{Statistical analysis}

All accelerometry outcome variables were computed for each individual day, then averaged over days for each valid participant using a 5:2 weighting for work/school days versus weekend days. School holidays were counted as weekend days for children.

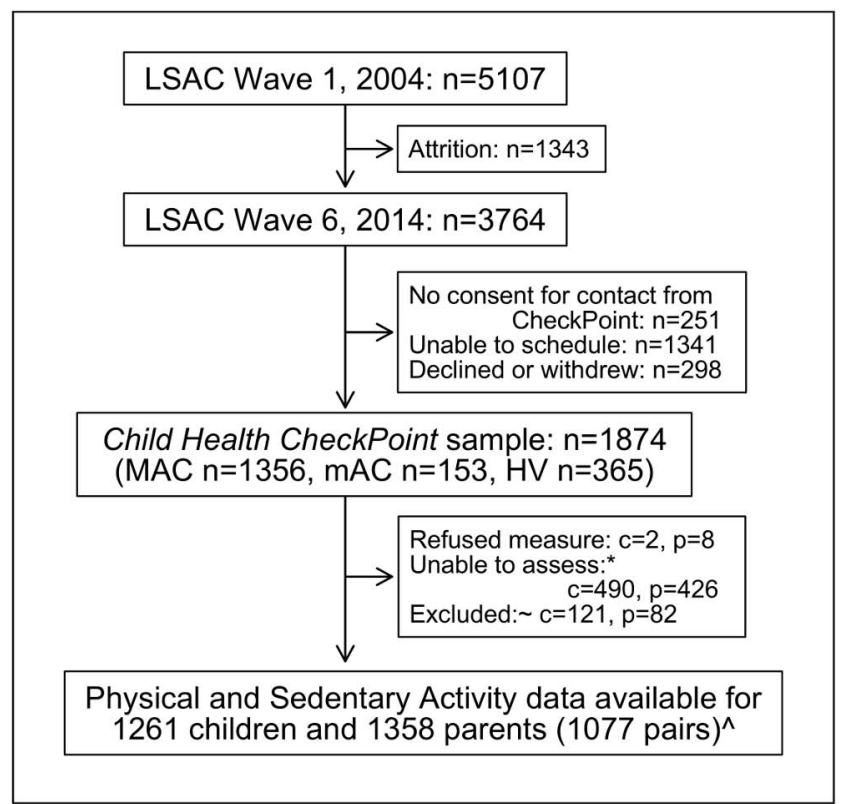

Figure 2 Recruitment and retention of participants for Child Health CheckPoint, including sample size for PA and SB. *Unable to assess due to equipment failure, poor quality data or time constraints. Participants excluded if valid days available did not meet the minimum criteria of at least 4 days of any type, $\leq 200 \mathrm{~min}$ sleep and $\leq 1000 \mathrm{~min}$ sedentary time. $\wedge$ Data from 12 non-biological child-parent pairs excluded from concordance analysis. n, number of families; c, number of children; HV, home visit assessment; LSAC, Longitudinal Study of Australian Children; MAC, main assessment centre; $\mathrm{mAC}$, mini assessment centre; $p$, number of attending adults; PA, physical activity; SB, sedentary behaviour .

Objective PA variables were described for all children and adults using means and SD. Population summary statistics were estimated by applying survey weights and survey procedures that corrected for sampling, participation and non-response biases and took into account clustering in the sampling frame. SEs were calculated taking into account the complex design and weights. More detail on the calculation of weights is provided elsewhere. ${ }^{53}$

For each of the 1077 biological child-parent pairs, concordance between parents and children was assessed by: (1) Pearson's correlation coefficients with 95\% CIs; and (2) linear regression with the child variable as the dependent variable and the parent variable as the independent variable. Linear regression models were adjusted for parent and child age and sex (in models including both sexes) and socioeconomic Disadvantage Index. As there were only minimal differences between unweighted and weighted results, only the former are presented here.

Data were analysed using Stata V.14.2.

\section{RESULTS}

\section{Sample characteristics}

Figure 2 shows that valid accelerometry data were obtained for 1261 children (50\% female) and 1358 parents, allowing the analysis of 1077 child-parent pairs. 
Table 1 Participant characteristics (weighted mean and SD)

\begin{tabular}{|c|c|c|c|c|c|c|c|c|c|}
\hline \multirow[b]{2}{*}{ Characteristic } & \multicolumn{3}{|l|}{ All } & \multicolumn{3}{|c|}{ Male } & \multicolumn{3}{|c|}{ Female } \\
\hline & $\mathbf{n}$ & mean & SD & $\mathbf{n}$ & mean & SD & $\mathbf{n}$ & mean & SD \\
\hline \multicolumn{10}{|l|}{ Child } \\
\hline BMI $\left(\mathrm{kg} / \mathrm{m}^{2}\right)$ & 1260 & 19.3 & 3.5 & 631 & 19.2 & 3.5 & 629 & 19.4 & 3.6 \\
\hline $\begin{array}{l}\text { Disadvantage } \\
\text { Index }\end{array}$ & 1257 & 1010 & 64 & 629 & 1010 & 63 & 628 & 1010 & 65 \\
\hline \multicolumn{10}{|l|}{ Parent } \\
\hline Age (years) & 1358 & 43.9 & 5.6 & 167 & 46.3 & 7.1 & 1191 & 43.6 & 5.3 \\
\hline BMI $\left(\mathrm{kg} / \mathrm{m}^{2}\right)$ & 1350 & 28.0 & 6.4 & 167 & 28.8 & 5.1 & 1183 & 27.9 & 6.5 \\
\hline
\end{tabular}

Sample sizes vary according to availability of data.

$\mathrm{BMI}$, body mass index; $\mathrm{n}$, sample size.

Only biological child-parent pairs were included in the concordance analysis, resulting in the exclusion of 12 non-biological pairs. Table 1 shows the participant characteristics. Most parents were mothers (88\%). Overall, participants were slightly more advantaged than the average Australian household, with a mean Disadvantage Index of about $0.1 \mathrm{SD}$ above the Australian average and a narrower spread (SD 64) than the national SD of 100. Body mass index (BMI) for parents and children were comparable with general population values for adults and children of the same age. ${ }^{54}$

\section{PA characteristics}

Table 2 presents the PA characteristics of all valid participants, including MVPA and SB duration and fragmentation, and VPA duration. Figure 3 shows the distributions of SB and MVPA duration for both parents and children. Parents had on average lower accelerometry counts than children (mean [SD] 209 [46] vs 284 [71] g.min). Overall, children accumulated an average of $32 \mathrm{~min}$ of MVPA per day, with boys having overall higher MVPA duration ( $40 \mathrm{~min} /$ day) than girls $(24 \mathrm{~min} /$ day). Variability (SD of the duration) was large for both boys and girls, relative to the mean (SD $30 \mathrm{~min}$ and $22 \mathrm{~min}$, respectively). Adults' MVPA duration was $142 \mathrm{~min}$ and $122 \mathrm{~min} /$ day for mothers and fathers, respectively, and the variability (SD 69 and $62 \mathrm{~min}$ for fathers and mothers, respectively) was lower than in children relative to the mean. Fifteen per cent of children and $93 \%$ of parents met MVPA recommendations of 60 and $30 \mathrm{~min} /$ day respectively. However, it is important to note that children's and parents' MVPA and SB durations are not directly comparable, because different cutpoints are used. Overall, children exhibited a more fragmented pattern of accumulation of MVPA (higher $\alpha$ ) than parents, with an overall average $\alpha$ of 2.00 for children and 1.85 for parents.

The sedentary time of boys and girls was averaging $679 \mathrm{~min}$ and $684 \mathrm{~min}$, respectively. This was larger than parents, with 555 and $538 \mathrm{~min}$ for fathers and mothers, respectively. Parents also exhibited a more fragmented accumulation of SB $(\alpha=2.46)$ than children $(\alpha=2.13)$.

\section{Child-parent concordance}

Table 3 presents the correlation coefficient (CC) and regression coefficient (RC) estimates between the children and their parents, for each of the five PA metrics. Overall, there were small but significant correlations between children's and parents' PA behaviours. Between mothers and children, all five PA variables were significantly correlated. Correlations were weak for SB, VPA and MVPA duration, and SB fragmentation, and very weak for MVPA fragmentation (0.11). Between fathers and children, only VPA duration and SB fragmentation were significantly associated $(\mathrm{r}=0.24$ and 0.22 , respectively, both $\mathrm{p}<0.05$ ); however, the sample size was smaller.

\section{Influence of MVPA imputation method}

Thirty-four per cent of children and $10 \%$ of parents had any amount of reported sport-related non-wear. Replacing these non-wear periods with MVPA may have had an influence on the results. To investigate this, we also replaced these sport-related non-wear periods with a composition of 50\% MVPA, 30\% LPA and 20\% sedentary time, as reported by Ridley et al in an observational study of children. ${ }^{55}$ Pearson's correlation between children's daily MVPA while using 100\% MVPA replacement, and using the $50 \% / 30 \% / 20 \%$ MVPA/LPA/sedentary composition, was 0.96 . Parent-child correlation for daily MVPA duration increased slightly from 0.16 (table 3 ) to 0.166 .

\section{DISCUSSION}

\section{Principal findings}

This study provides normative values for device-measured activity behaviour in a large sample of Australian midlife adults and children aged 11-12years. Using the specific combination of device and analytical algorithms in this study, children accumulated on average $32 \mathrm{~min}$ of MVPA each day, of which $10 \mathrm{~min}$ were VPA. Using different cutpoints, adults accumulated $125 \mathrm{~min} /$ day of MVPA, but only $7 \mathrm{~min} /$ day of VPA. Children had higher levels of sedentary time $(681 \mathrm{~min} /$ day $)$ than adults $(540 \mathrm{~min} /$ day). Children's MVPA was more fragmented than that of 


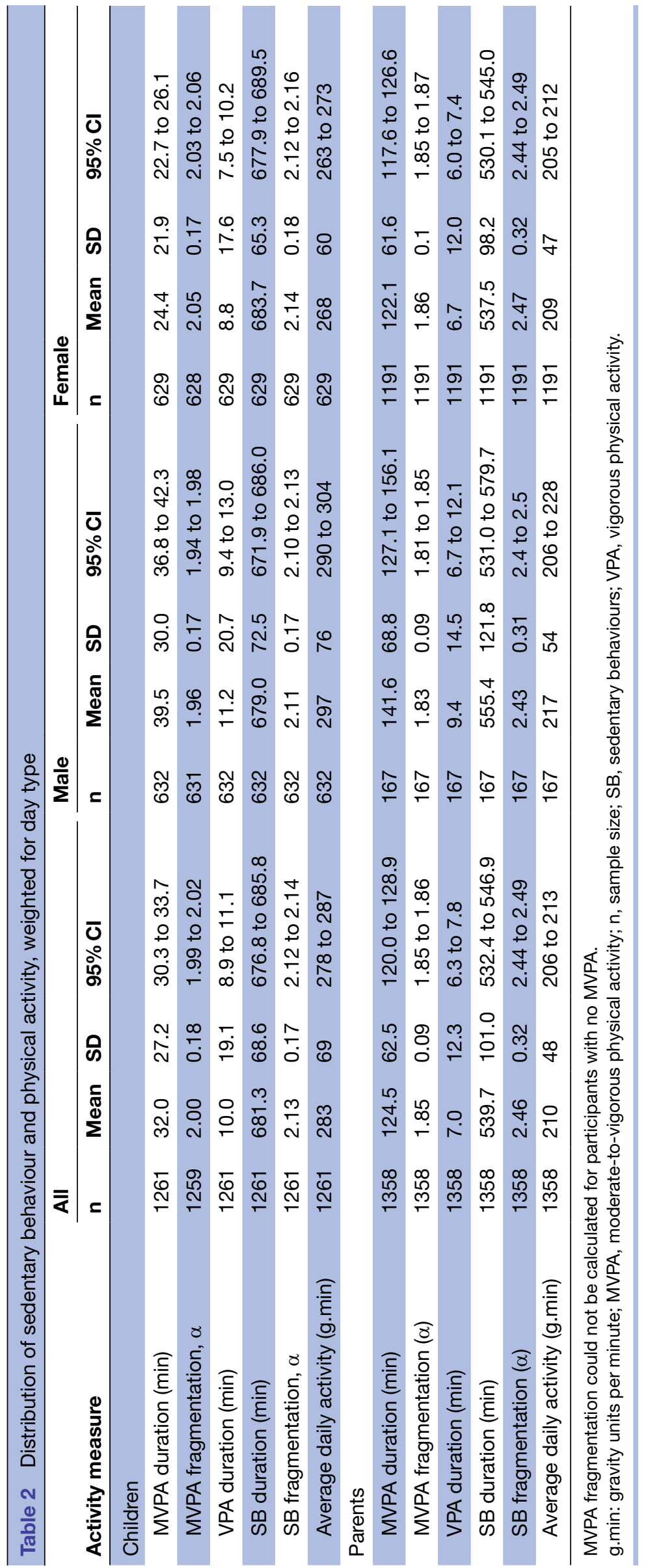



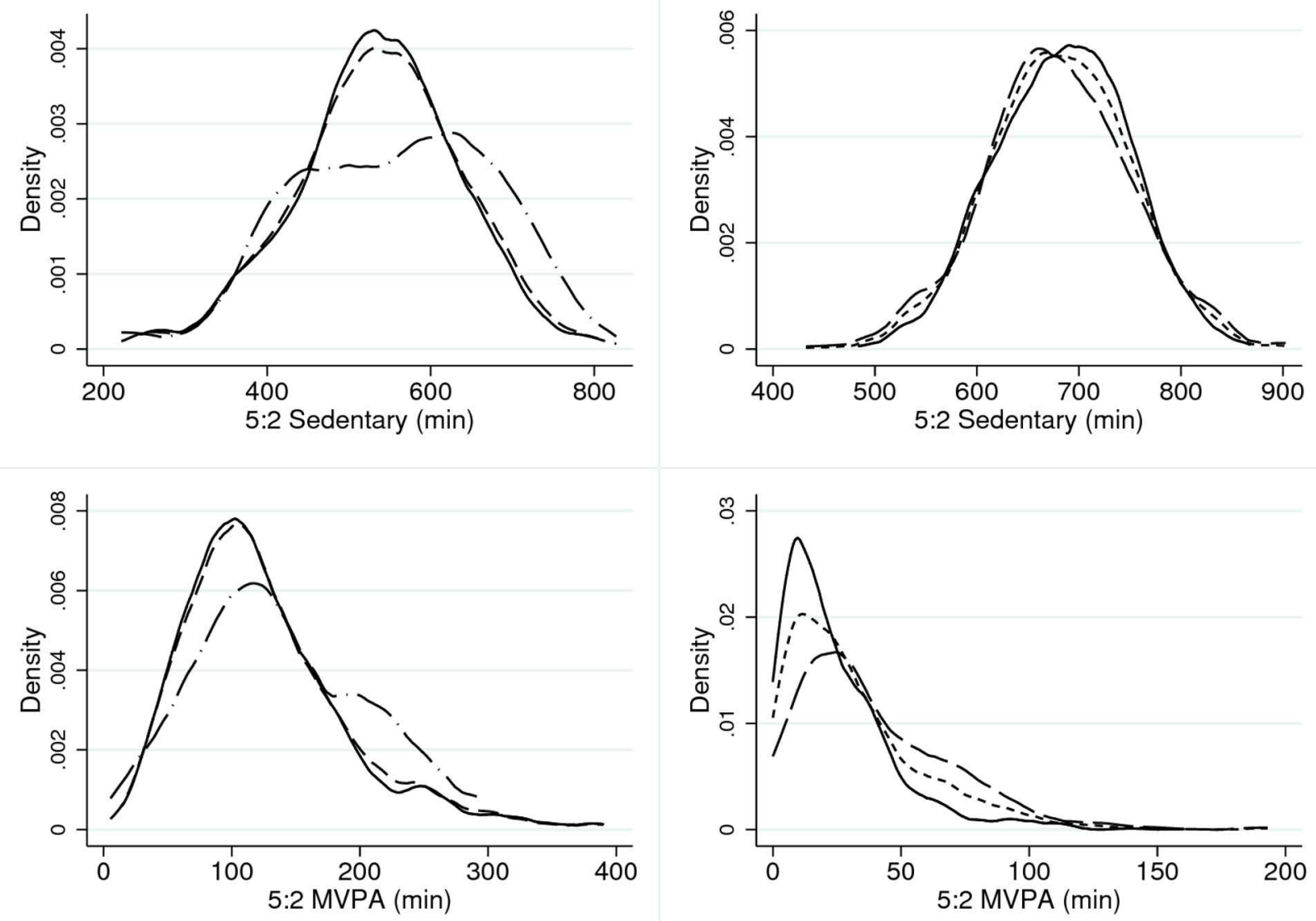

Figure 3 Density plots for average sedentary and MVPA time per day. Left-hand plots relate to parents, right-hand plots relate to children. Males/boys (dash-dotted line), females/girls (solid line) and both sexes combined (dotted line). MVPA, moderate-tovigorous physical activity.

Table 3 Parent-child concordance. The upper panel shows unadjusted values, and the lower panel values adjusted for parent and child age and sex, and Disadvantage Index.

\begin{tabular}{|c|c|c|c|c|c|c|c|c|c|}
\hline \multirow[b]{2}{*}{ Pearson's correlation } & \multicolumn{3}{|c|}{ Parent-child } & \multicolumn{3}{|c|}{ Father-child } & \multicolumn{3}{|c|}{ Mother-child } \\
\hline & $\mathbf{n}$ & CC & $95 \% \mathrm{Cl}$ & $\mathbf{n}$ & CC & $95 \% \mathrm{Cl}$ & $\mathbf{n}$ & CC & $95 \% \mathrm{Cl}$ \\
\hline MVPA duration (min) & 1077 & 0.16 & 0.11 to 0.22 & 128 & 0.13 & -0.05 to 0.29 & 949 & 0.17 & 0.11 to 0.23 \\
\hline MVPA fragmentation $(\alpha)$ & 1076 & 0.10 & 0.04 to 0.16 & 128 & 0.04 & -0.13 to 0.22 & 948 & 0.11 & 0.05 to 0.18 \\
\hline VPA duration (min) & 1077 & 0.19 & 0.14 to 0.25 & 128 & 0.24 & 0.07 to 0.40 & 949 & 0.19 & 0.13 to 0.25 \\
\hline SB duration (min) & 1077 & 0.16 & 0.11 to 0.22 & 128 & 0.13 & -0.05 to 0.29 & 949 & 0.17 & 0.11 to 0.24 \\
\hline SB fragmentation $(\alpha)$ & 1077 & 0.16 & 0.10 to 0.22 & 128 & 0.22 & 0.05 to 0.38 & 949 & 0.15 & 0.09 to 0.21 \\
\hline $\begin{array}{l}\text { Linear Regression } \\
\text { (adjusted for covariates) }\end{array}$ & $\mathbf{n}$ & $\mathbf{R C}$ & $P$ value & $\mathbf{n}$ & $\mathbf{R C}$ & $P$ value & $\mathbf{n}$ & $\mathbf{R C}$ & $P$ value \\
\hline MVPA duration (min) & 1076 & 0.32 & $<0.001$ & 127 & 0.20 & 0.24 & 949 & 0.34 & $<0.001$ \\
\hline MVPA fragmentation $(\alpha)$ & 1075 & 0.20 & 0.001 & 127 & 0.04 & 0.84 & 948 & 0.22 & 0.001 \\
\hline VPA duration (min) & 1076 & 0.23 & $<0.001$ & 127 & 0.27 & 0.03 & 949 & 0.23 & $<0.001$ \\
\hline SB duration (min) & 1076 & 0.11 & $<0.001$ & 127 & 0.10 & 0.07 & 949 & 0.12 & 0.001 \\
\hline SB fragmentation $(\alpha)$ & 1076 & 0.08 & $<0.001$ & 127 & 0.13 & 0.01 & 949 & 0.08 & $<0.001$ \\
\hline
\end{tabular}

Covariates in adjusted linear regression models include parent and child age and sex, and Disadvantage Index. MVPA fragmentation could not be calculated for participants with no MVPA.

CC, correlation coefficient; MVPA, moderate-to-vigorous physical activity; n: sample size; RC, estimated regression coefficient; SB, sedentary behaviours; VPA, vigorous physical activity. 
their parents, while sedentary time was less fragmented. Concordance between children and parents for MVPA, VPA and sedentary time duration and fragmentation was weak to moderate, ranging between $r=0.08$ and $r=0.32$.

\section{Strengths and limitations}

The CheckPoint PA study is the largest accelerometry-based cross-generational study of activity in Australia. It is also the first to report concordance of PA and SBs between parents and children aged 11-12 years. This study reports valid, reliable, objective, free-living measures of child and parent PA patterns and their concordance from a large national sample. Data were collected simultaneously for parents and children using the same protocol. To our knowledge, this is the first study to report metrics for intensity and fragmentation of PA and SB.

Limitations include the relatively small number of fathers, reducing precision of their estimates. Only one parent was included for each child; this is nonetheless one of very few studies that present separate (but largely similar) mother-child and father-child concordance estimates. Second, the sample in this study, while drawn from a cohort which was designed to be nationally representative, was subject both to selective update and attrition. This might have affected both activity and the impacts of BMI on activity, and perhaps concordance values. Furthermore, the age range of the children was narrow (11-12 years).

\section{Significance and meaning}

\section{Duration of PA and sedentary time}

It is surprising that our results indicate parents accumulated more daily MVPA than children (table 2). Most studies find that children are more active than adults, both by self-report and using accelerometers. ${ }^{14}$ Moreover, doubly labelled water studies show that TDEE declines with age. ${ }^{56}$ In that regard, the differences in this study between children and adults almost certainly come from the fact that two separate sets of cutpoints were used. Although the cutpoints we used were developed specifically for children and adults, the MPA cutpoint for children (788g.min) is almost double that of adults ( $403 \mathrm{~g}$. min), resulting in a much lower MVPA estimate for children. The fact that daily average accelerations were $35 \%$ higher for children than parents (283 g.min and $210 \mathrm{~g}$. min, respectively) confirms the fact that children in fact moved more than their parents, and children exhibiting lower daily MVPA is an artefact of the different cutpoints used. In other words, using children's cutpoints to analyse parents' MVPA would result in parents exhibiting less daily MVPA than children.

In spite of this, estimated MVPA and sedentary durations in the CheckPoint study were in line with findings from several previous studies. For instance, in adults, Rosenberger et a $\tilde{l}^{\tilde{7}}$ reported MVPA durations of 100-220 min/ day depending on the device used. Recently, Rowlands $e t$ $a \bar{l}^{58}$ reported average MVPA durations of $92 \mathrm{~min} /$ day for a small adult sample, using the GeneActiv. A recent study using the large $(n=22978)$ Biobank dataset reported $106 \mathrm{~min} /$ day MVPA in healthy adults. ${ }^{59}$ In children, Telford et $a l^{60}$ reported averages of 43 and $31 \mathrm{~min} /$ day of MVPA for boys and girls aged 8-12 years, respectively. The International Study of Childhood Obesity, Lifestyle and Environment (ISCOLE) study used Actigraph GT3X+ accelerometers, finding that 10 -year-old boys accumulated $75 \mathrm{~min} /$ day MVPA and girls $57 \mathrm{~min} /$ day. ${ }^{61}$

Of note, accelerometer-based estimates of children's PA tend to show more variability between studies than adults' PA. This may be due, in part, to the more sporadic nature of children's PA. A study of 47 children aged 6-11 by Schaefer $e t a l^{62}$ showed that daily MVPA was $308 \mathrm{~min} /$ day when including all episodes of $1 \mathrm{~s}$ or more, but decreased 20-fold, to $14 \mathrm{~min} /$ day, when only including MVPA episodes of at least $60 \mathrm{~s}$. Second, a study by Reilly et al showed that estimates of children's MVPA varied from $28 \mathrm{~min} /$ day to $266 \mathrm{~min} /$ day depending on the set of cutpoints used. ${ }^{63}$

Overall, our results once again highlight the fact that accelerometry-based assessment of PA is highly dependent on a number of factors, including the accelerometer device itself $^{58}$ but more importantly the choice of algorithms and processes used, such as sampling frequency, raw data filtering, epoch length and cutpoint values. At present, there is no consensus on the choice of processes. ${ }^{64}$ A recent review has identified wide discrepancies in processing parameters, ${ }^{49}$ with some of these choices having a large impact on results. This makes comparison between studies difficult. In this regard, accelerometry could be seen as better suited to assess relative change in $\mathrm{PA}$, be it across populations, in longitudinal studies or in interventions, provided that protocols and data processing methods are consistent. Assessment of absolute values of PA appear more difficult since they are highly dependent on a number of factors. This also means it is challenging to use objectively measured PA to decide whether children and adults meet PA guidelines, which were themselves originally developed based on self-report, rather than accelerometry. While most PA guidelines ${ }^{10116365}$ recommend $60 \mathrm{~min}$ and $30 \mathrm{~min}$ per day for children adults, respectively, assessing guideline adherence using accelerometry is inherently limited. Using our combination of device and algorithms, only $15 \%$ of children meet these recommendations. A recent Australian survey showed that only $19 \%$ of children aged 5-17 years meet the PA guidelines. ${ }^{66}$ Our study indicates that Australian adults easily met the recommendations, which is at odds with self-report data. ${ }^{67}$

\section{Fragmentation of PA and sedentary time}

Parents presented fragmentation of sedentary time (2.46) in line with levels of healthy populations (2.27).$^{52}$ Children's sedentary fragmentation was overall lower than adults', perhaps reflecting both long periods of sitting during school and sessions of unbroken screen time. Children spent $50 \%$ of their sedentary time in bouts of $43 \mathrm{~min}$ or more and parents in bouts of $26 \mathrm{~min}$ or more. 
In contrast, children exhibited a more fragmented accumulation of MVPA than adults, in line with observations that children's PA tends to be sporadic. ${ }^{68} 69$ Our study is the first to use the alpha coefficient to quantify fragmentation of MVPA in adults, and the first to use it for both sedentary time and MVPA in children, so there is no available comparison point.

\section{Concordance between children and parents}

The correlation for MVPA duration $(\mathrm{r}=0.16)$ was consistent with our review of studies $(r=0.15-0.21)$, most of which used self-report. The method chosen for imputing MVPA time during sport-related non-wear events only had little effect. The weakest correlation was for MVPA fragmentation $(\mathrm{r}=0.10)$ and the strongest for VPA duration $(\mathrm{r}=0.19)$. This suggests that genetic effects on objective MVPA and SB are relatively modest and that non-shared environments (principally work and school) may be the major determinant.

\section{Implications for clinicians and policy makers}

Children's MVPA levels in this study were low (32 min/ day), and sedentary time was high $(681 \mathrm{~min} /$ day $)$ and showed evidence or prolonged, unbroken sedentary periods. Australian children are not sufficiently active, and efforts should be made to increase participation. Regarding sedentary time, we found that children accumulate more than 11 hours/day of sedentary time. However, the guidelines generally provide recommendations regarding screen time only, ${ }^{10} 1165$ whereas accelerometers do not distinguish screen time from the rest of sedentary time. A previous study of Australian children ${ }^{70}$ found that this high level of sedentary time represents mainly screen time $(40 \%)$, sitting at school (25\%), sedentary social occasions $(12 \%)$, eating $(10 \%)$ and passive transport $(10 \%)$. While interventions can address each of these domains, there is mounting evidence that not all types of sedentary time are equally harmful, with television in particular being inculpated in unfavourable health outcomes. The composition of sedentary time may therefore be as important as the overall duration.

For adults, the vast majority meet the recommended MVPA. Regarding sedentary time, guidelines tend to only recommend that sedentary time should be broken up into bouts of $30 \mathrm{~min}$ or less when possible. In their study, Chastin $e t a \tilde{l}^{2}$ showed that a fragmentation index of 2.27 means that half the total sedentary time is accumulated in bouts of $17 \mathrm{~min}$ or shorter. Our results for adults $(\alpha=2.46)$ mean that the sedentary time for our adult sample is even more fragmented that this, indicating sufficient fragmentation of sedentary time. That said, the extent to which sedentary time must be broken down in order to avoid negative health effects is unknown.

\section{Unanswered questions and future research}

This study highlights the need to standardise methods for objective measures of use of time to enable pooling and comparison of results from different countries and study centres and across ages. Moreover, there is a need to enable better comparisons between objectively measured PA and guidelines based on subjective reports. One initiative might be to establish a repository of raw accelerometry data, along with harmonised key covariates, which can be reanalysed using common metrics. The International Children's Accelerometry Database is moving towards this objective. ${ }^{71}$

While the cross-sectional nature of the study does not allow us to infer causation, the relatively weak concordance values suggest that genetic factors relating to PA and sedentary time are not strong and that parents only moderately influence their children's PA and SBs (and/ or vice versa). The latter is to be expected given that children spend a large part of their waking day at school. The relative contribution of genetic and environmental factors could be addressed by genetic studies using, for example, Mendelian randomisation.

Acknowledgements This paper uses unit record data from Growing Up in Australia, the Longitudinal Study of Australian Children. The study is conducted in partnership between the Department of Social Services (DSS), the Australian Institute of Family Studies (AIFS) and the Australian Bureau of Statistics (ABS). Research Electronic Data Capture (REDCap) electronic data capture tools were used in this study. More information about this software can be found at: http://www. project-redcap.org. The authors would like to thank the LSAC and CheckPoint study participants, staff and students for their contributions.

Contributors FF, JM, MW and TO conceptualised the manuscript. FF led the writing; ACG, JM, MW and TO provided expert advice and critical review of this manuscript; ACG analysed the data. MW is the principal investigator of the Child Health CheckPoint, planned the analyses and provided critical review of this manuscript.

Funding This work was supported by the National Health and Medical Research Council (NHMRC) of Australia (Project Grants 1041352 and 1109355), The Royal Children's Hospital Foundation (2014-241), the Murdoch Children's Research Institute (MCRI), The University of Melbourne, the National Heart Foundation of Australia (100660) and Financial Markets Foundation for Children (2014-055 and 2016-310). MW was supported by Australian NHMRC Senior Research Fellowship 1046518 and Cure Kids New Zealand. The MCRI administered the research grants for the study and provided infrastructural support (IT and biospecimen management) to its staff and the study but played no role in the conduct or analysis of the trial. The Department of Social Services played a role in study design; however, no other funding bodies had a role in the study design and conduct; data collection, management, analysis and interpretation; preparation, review or approval of the manuscript; and decision to submit the manuscript for publication. Research at the MCRI is supported by the Victorian Government's Operational Infrastructure Support Program.

Disclaimer The findings and views reported in this paper are those of the author and should not be attributed to DSS, AIFS or the ABS.

Competing interests All authors have completed the ICMJE uniform disclosure form at http://www.icmje.org/coi_disclosure.pdf and declare financial support as described in the funding section. MW received support from Sandoz to present at a symposium outside the submitted work.

Patient consent for publication Not required.

Ethics approval The CheckPoint study protocol was approved by The Royal Children's Hospital (Melbourne, Australia) Human Research Ethics Committee (33225D) and Australian Institute of Family Studies Ethics Committee (14-26).

Provenance and peer review Not commissioned; externally peer reviewed.

Data sharing statement The Longitudinal Study of Australian Children datasets and technical documents are available to researchers at no cost via a licence agreement. Data access requests are co-ordinated by the National Centre for Longitudinal Data. More information is available at https://dataverse.ada.edu.au/ dataverse/lsac. 
Open access This is an open access article distributed in accordance with the Creative Commons Attribution Non Commercial (CC BY-NC 4.0) license, which permits others to distribute, remix, adapt, build upon this work non-commercially, and license their derivative works on different terms, provided the original work is properly cited, appropriate credit is given, any changes made indicated, and the use is non-commercial. See: http://creativecommons.org/licenses/by-nc/4.0/.

\section{REFERENCES}

1. Janssen I, Leblanc AG. Systematic review of the health benefits of physical activity and fitness in school-aged children and youth. Int $J$ Behav Nutr Phys Act 2010;7:40.

2. Tremblay MS, LeBlanc AG, Kho ME, et al. Systematic review of sedentary behaviour and health indicators in school-aged children and youth. Int J Behav Nutr Phys Act 2011;8:98.

3. Pate RR, Pratt M, Blair SN, et al. Physical activity and public health. A recommendation from the Centers for Disease Control and Prevention and the American College of Sports Medicine. JAMA 1995;273:402-7.

4. Thorp AA, Owen N, Neuhaus M, et al. Sedentary behaviors and subsequent health outcomes in adults a systematic review of longitudinal studies, 1996-2011. Am J Prev Med 2011;41:207-15.

5. Healy GN, Dunstan DW, Salmon J, et al. Breaks in sedentary time. Diabetes care 2008;31:661-6.

6. Saunders TJ, Tremblay MS, Mathieu MÈ, et al. Associations of sedentary behavior, sedentary bouts and breaks in sedentary time with cardiometabolic risk in children with a family history of obesity. PLoS One 2013;8:e79143.

7. Gutin B, Barbeau P, Owens S, et al. Effects of exercise intensity on cardiovascular fitness, total body composition, and visceral adiposity of obese adolescents. Am J Clin Nutr 2002;75:818-26.

8. Lee IM, Sesso HD, Oguma Y, et al. The "weekend warrior" and risk of mortality. Am J Epidemiol 2004;160:636-41.

9. Bull F, Biddle S, Buchner D, et al. Physical activity guidelines in the UK: review and recommendations: School of Sport, Exercise and Health Sciences, Loughborough University, 2010.

10. World Health Organization. Global recommendations on physical activity for health, 2010

11. Tremblay MS, Warburton DE, Janssen I, et al. New Canadian physical activity guidelines. Appl Physiol Nutr Metab 2011;36:36-46.

12. Fisher A, Smith $L$, van Jaarsveld $\mathrm{CH}$, et al. Are children's activity levels determined by their genes or environment? A systematic review of twin studies. Prev Med Rep 2015;2:548-53.

13. Colley RC, Garriguet D, Janssen I, et al. Physical activity of Canadian children and youth: accelerometer results from the 2007 to 2009 Canadian Health Measures Survey. Health Rep 2011;22:15.

14. Troiano RP, Berrigan D, Dodd KW, et al. Physical activity in the United States measured by accelerometer. Med Sci Sports Exerc 2008;40:181-8.

15. Colley RC, Garriguet D, Janssen I, et al. Physical activity of Canadian adults: accelerometer results from the 2007 to 2009 Canadian Health Measures Survey. Health Rep 2011;22:7.

16. O'Donovan $\mathrm{G}$, Hillsdon M, Ukoumunne OC, et al. Objectively measured physical activity, cardiorespiratory fitness and cardiometabolic risk factors in the Health Survey for England. Prev Med 2013;57:201-5

17. Zecevic CA, Tremblay L, Lovsin T, et al. Parental Influence on Young Children's Physical Activity. Int J Pediatr 2010;2010:1-9.

18. Jacobi D, Caille A, Borys JM, et al. Parent-offspring correlations in pedometer-assessed physical activity. PLoS One 2011;6:e29195.

19. Jago R, Fox KR, Page AS, et al. Parent and child physical activity and sedentary time: do active parents foster active children? BMC Public Health 2010;10:194.

20. Sijtsma A, Sauer PJ, Corpeleijn E. Parental correlations of physical activity and body mass index in young children--The GECKO Drenthe cohort. Int J Behav Nutr Phys Act 2015;12:132.

21. Fogelholm M, Nuutinen $\mathrm{O}$, Pasanen $\mathrm{M}$, et al. Parent-child relationship of physical activity patterns and obesity. Int J Obes Relat Metab Disord 1999;23:1262-8.

22. Fuemmeler BF, Anderson CB, Mâsse LC. Parent-child relationship of directly measured physical activity. Int J Behav Nutr Phys Act 2011;8:17.

23. Moore LL, Lombardi DA, White MJ, et al. Influence of parents physical activity levels on activity levels of young children. J Pediatr 1991;118:215-9.

24. Dong F, Howard AG, Herring A, et al. Abstract P281: Longitudinal Associations of Diet, Screen Time, and Physical Activity Behaviors With Cardiometabolic Risk Factors Among Chinese Children and Their Parents: Am Heart Assoc, 2017.
25. Pan C-Y, Frey GC, Bar-Or O, et al. Concordance of Physical Activity Among Parents and Youth with Physical Disabilities. J Dev Phys Disabil 2005;17:395-407.

26. Rossow I, Rise J. Concordance of parental and adolescent health behaviors. Soc Sci Med 1994;38:1299-305.

27. Loucaides CA, Jago R. Correlates of pedometer-assessed physical activity in cypriot elementary school children. J Phys Act Health 2006;3:267-76.

28. Eriksson M, Nordqvist T, Rasmussen F. Associations between parents' and 12-year-old children's sport and vigorous activity: the role of self-esteem and athletic competence. J Phys Act Health 2008;5:359-73.

29. Godin G, Shephard RJ. Psychosocial factors influencing intentions to exercise of young students from grades 7 to 9. Res Q Exerc Sport 1986;57:41-52.

30. Runyan SM, Stadler DD, Bainbridge CN, et al. Familial resemblance of bone mineralization, calcium intake, and physical activity in earlyadolescent daughters, their mothers, and maternal grandmothers. $J$ Am Diet Assoc 2003;103:1320-5.

31. Dempsey JM, Kimiecik JC, Horn TS. Parental Influence on Children's Moderate to Vigorous Physical Activity Participation: An ExpectancyValue Approach. Pediatr Exerc Sci 1993;5:151-67.

32. McMinn AM, van Sluijs EM, Wedderkopp N, et al. Sociocultural correlates of physical activity in children and adolescents: findings from the Danish arm of the European Youth Heart study. Pediatr Exerc Sci 2008;20:319-32.

33. Raudsepp L, Viira R. Sociocultural correlates of physical activity in adolescents. Pediatr Exerc Sci 2000;12:51-60.

34. Xiao Lin Yang, Telama R, Laakso L. Parents' physical activity, socioeconomic status and education as predictors of physical activity and sport among children and youths - a 12-year follow-up study. Int Rev Sociol Sport 1996;31:273-91.

35. Sallis JF, Nader PR, Broyles SL, et al. Correlates of physical activity at home in mexican-american and anglo-american preschool children. Health Psychol 1993;12:390-8.

36. Gottlieb NH, Chen MS. Sociocultural correlates of childhood sporting activities: their implications for heart health. Soc Sci Med 1985;21:533-9.

37. McGuire MT, Hannan PJ, Neumark-Sztainer D, et al. Parental correlates of physical activity in a racially/ethnically diverse adolescent sample. J Adolesc Health 2002;30:253-61.

38. Epstein LH, Paluch RA, Coleman KJ, et al. Determinants of physical activity in obese children assessed by accelerometer and self-report. Med Sci Sports Exerc 1996;28:1157-64.

39. Pahkala K, Heinonen OJ, Lagström $\mathrm{H}$, et al. Leisure-time physical activity of 13-year-old adolescents. Scand J Med Sci Sports 2007:17:324-30.

40. Freedson PS, Evenson S. Familial aggregation in physical activity. Res Q Exerc Sport 1991;62:384-9.

41. Sanson A, Johnstone R. 'Growing Up in Australia' takes its first steps. Family Matters 2004;67:46-53.

42. Edwards B. Growing Up in Australia: the Longitudinal Study of Australian Children: entering adolescence and becoming a young adult. Family Matters 2014;95:5-14.

43. Australian Institute of Family Studies. Survey methodology: The Longitudinal Study of Australian Children data user guide. 2015 http://www.growingupinaustralia.gov.au/data/docs/userguide/11 surveymethodology.html (Accessed 9 Mar 2018).

44. Australian Institute of Family Studies. Growing Up in Australia: The Longitudinal Study of Australian Children. 2015 http://www. growingupinaustralia.gov.au/ (Accessed 9 Mar 2018).

45. Australian Institute of Family Studies. About Growing Up in Australia. 2015. http://www.growingupinaustralia.gov.au/about/index.html (Accessed 9 Mar 2018).

46. Clifford SA, Davies S, Wake M. Child Health Checkpoint: Cohort summary and methodology of a physical health and biospecimen module for the Longitudinal Study of Australian Children. BMJ Open 2019;9(suppl 3):3-22.

47. Wake M, Clifford SA, York E, et al. Introducing Growing Up in Australia's Child Health Checkpoint: A physical health and biomarkers module for the Longitudinal Study of Australian Children. Family Matters 2014;95:15-23.

48. Davies S, Clifford SA, Gillespie AN, et al. Longitudinal Study of Australian Children's Child Health Checkpoint Data Issues Paper December 2018. Melbourne: Murdoch Children's Research Institute, 2018.

49. Migueles $\mathrm{JH}$, Cadenas-Sanchez $\mathrm{C}$, Ekelund U, et al. Accelerometer data collection and processing criteria to assess physical activity and other outcomes: A systematic review and practical considerations. Sports Med 2017;47:1821-45. 
50. Esliger DW, Rowlands AV, Hurst TL, et al. Validation of the GENEA Accelerometer. Med Sci Sports Exerc 2011;43:1085-93.

51. Phillips LR, Parfitt G, Rowlands AV. Calibration of the GENEA accelerometer for assessment of physical activity intensity in children. J Sci Med Sport 2013;16:124-8.

52. Chastin SF, Granat MH. Methods for objective measure, quantification and analysis of sedentary behaviour and inactivity. Gait Posture 2010;31:82-6.

53. Ellul S, Hiscock R, Mensah F, et al. Longitudinal Study of Australian Children's Child Health CheckPoint Technical Paper 1: Weighting and Non-Response. Melbourne: Murdoch Children's Research Institute, 2018.

54. Australian Health Survey: updated results, overweight and obesity (2011-2012). Canberra: Australian Government, 2013.

55. Ridley K, Zabeen S, Lunnay BK. Children's physical activity levels during organised sports practices. J Sci Med Sport 2018;21:930-4.

56. Goran MI, Poehlman ET, Johnson RK. Energy requirements across the life span: New findings based on measurement of total energy expenditure with doubly labeled water. Nutr Res 1995;15:115-50.

57. Rosenberger ME, Buman MP, Haskell WL, et al. Twenty-four hours of sleep, sedentary behavior, and physical activity with nine wearable devices. Med Sci Sports Exerc 2016;48:457-65

58. Rowlands AV, Yates T, Davies M, et al. Raw accelerometer data analysis with ggir r-package: Does accelerometer brand matter? Med Sci Sports Exerc 2016;48:1935-41.

59. Cassidy S, Fuller H, Chau J, et al. Accelerometer-derived physical activity in those with cardio-metabolic disease compared to healthy adults: a UK Biobank study of 52,556 participants. Acta Diabetol 2018;55:975-9.

60. Telford RM, Telford RD, Cunningham RB, et al. Longitudinal patterns of physical activity in children aged 8 to 12 years: the LOOK study. Int J Behav Nutr Phys Act 2013;10:81.
61. Katzmarzyk PT, Barreira TV, Broyles ST, et al. Relationship between lifestyle behaviors and obesity in children ages 9-11: Results from a 12-country study. Obesity 2015;23:1696-702.

62. Schaefer CA, Nigg CR, Hill JO, et al. Establishing and evaluating wrist cutpoints for the GENEActiv accelerometer in youth. Med Sci Sports Exerc 2014;46:826-33.

63. Reilly JJ, Penpraze V, Hislop J, et al. Objective measurement of physical activity and sedentary behaviour: review with new data. Arch Dis Child 2008;93:614-9.

64. Cain KL, Sallis JF, Conway TL, et al. Using accelerometers in youth physical activity studies: a review of methods. J Phys Act Health 2013;10:437-50.

65. Australia's Physical Activity and Sedentary Behaviour Guidelines. Canberra: Commonwealth of Australia, 2014.

66. Australian Health Survey: physical activity (2011-2012). Canberra: Australian Government, 2013.

67. Devonshire-Gill KR, Norton KI. Australian Adult Physical Activity Sufficiency Trend Data: Positive, Prevalent, and Persistent Changes 2002-2012. Journal of Physical Activity and Health 2017;20:1-10.

68. Corbin CB, Pangrazi RP. Physical activity for children: A statement of guidelines: NASPE Publications, 1998.

69. Strong WB, Malina RM, Blimkie CJ, et al. Evidence based physical activity for school-age youth. J Pediatr 2005;146:732-7.

70. Olds TS, Maher CA, Ridley K, et al. Descriptive epidemiology of screen and non-screen sedentary time in adolescents: a cross sectional study. Int J Behav Nutr Phys Act 2010;7:92.

71. Cooper AR, Goodman A, Page AS, et al. Objectively measured physical activity and sedentary time in youth: the International children's accelerometry database (ICAD). Int J Behav Nutr Phys Act 2015;12:113. 